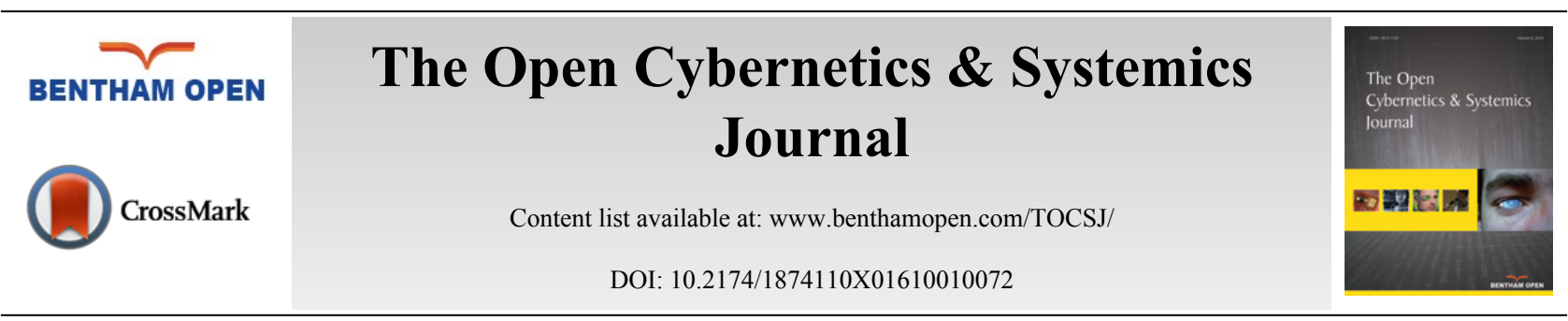

\title{
Study for Dynamic Computational Method of User Reliability in Open Knowledge Organization
}

\author{
Lanping Feng ${ }^{1,2, *}$, Fengping $\mathrm{Wu}^{1,2}$, Hanbing $\mathrm{Jin}^{1}$ and Zhen $\mathrm{Shi}^{1}$ \\ ${ }^{I}$ Information Management Department of Hohai University, Changzhou, 213022, PR China \\ ${ }^{2}$ Business School of Hohai University, Nanjing, 210098, PR China
}

Received: March 4, 2015

Revised: October 2, 2015

Accepted: October 5, 2015

\begin{abstract}
For the existing problem of user reliability computing in open knowledge organization, the current related researches are analyzed, and then this paper puts forward a dynamic computational method of user reliability based on multi-attribute evaluation. The method introduces direct reliability, bonus-penalty factor, group consumption factor and user consumption factor to compute the user reliability. Followed by stochastic simulation experiments after all, compared to the existing models, this method is able to control the low quality behavior of users, and fully and dynamically reflect the influence of the behavior of the user and the group on user reliability. In addition, it gives a better picture of user reliability from multiple perspectives.
\end{abstract}

Keywords: Dynamic computational method, Multi-attribute evaluation, Open knowledge organization, User reliability.

\section{INTRODUCTION}

With the development of Web technology, open knowledge organization method has become an important topic in the field of academia and industry [1]. In open knowledge organization system, a knowledge organizer plays the role of an ordinary user, who is not only knowledge consumer but also a provider, and participates in knowledge organization autonomously and freely, which improves the efficiency of knowledge organization and speeds up the knowledge updation greatly so as to make the users more satisfied. Nevertheless, ordinary users are mainly from the web, the knowledge that they provide has different qualities and quantities, only a small number of users provide most useful knowledge [2, 3], and users take advantage of this knowledge, which is mainly based on mutual trust [4 - 8]. Therefore, it is necessary for a method to quantify the trust of other users on a user into the user reliability, based on which users or applications can judge the knowledge correctness provided by ordinary users, and find domain users with professional knowledge and domain authority who plays very well the role of an expert in open knowledge organization.

At present, the representative open knowledge organization system is network encyclopedia, mainly computing user reliability from the perspective of user contribution behavior. For example, Baidu encyclopedia expresses user reliability from the aspect of user behavior such as behavior integral and the entry pass rate [9], and Wikipedia ranks users by voting based on user contribution [8]. Both of them do not consider the influence of user's own behavior and group behavior in same domain on user reliability. However, in the network encyclopedia system, the mutual influence among the users cannot be ignored [10]; the user behavior is diverse and complex, involves multiple domains, and has dynamic, time-varying, uncertain characteristic. Therefore, it is necessary to consider variety of key attributes to compute the reliability of user behavior from multiple perspectives.

Based on multi-attribute evaluation, this paper proposes a user reliability dynamic computational method (URDCM) to express the user reliability from the perspective of direct reliability, group consumption factor, user consumption

\footnotetext{
* Address correspondence to this author at the Department of Information Management, Hohai University; E-mails: fenglp@hhuc.edu.cn and fenglp1996@163.com
} 
factor and bonus-penalty factor. Stochastic simulation will be used to simulate the dynamic evolution of the user reliability, and the influence of the group and user's own behavior on his reliability will be discussed, so as to promote the positivity of users in knowledge exchanging, sharing and knowledge organizing, and to improve the efficiency and quality of knowledge organization.

\section{RELATED WORK}

At present, researches of user behavior reliability mainly focus on electronic business, network security and other domains. They mainly study how to quantify the reliability of network user behavior, and more accurately and fully express the influence of user behavior and its changes on the user reliability based on multi-attribute. For example, Li Xiaoyong and Zhang Shaogang et al. introduced multiple decision attributes to quantify the dynamic trust relationship between entities, from the views of the direct trust, risk, historical trust and feedback trust, and more finely characterize complexity and uncertainty of the trust [11]. Song Lingling et al. studied the algorithm of user evaluation reliability in service reputation computation from the user's subjective evaluation [12]. Lu You et al. proposed an adaptive userbehavior-evaluation method based on network status introducing the rough set theory [13]. Liu Qihong and Wu Xiaonian et al. presented a weighted trust computation method based on the behavior of users, which accurately and dynamically reflect the users-behavioral changes and the time attribute of feedback information, direct trust and recommendation trust are important indexes [14]. From the perspectives of direct and indirect reliability, and behavior positive degree, Jiang Ze et al. put forward the evaluation model of behavior reliability of network user based on multiattribute decision [15]. Lu You et al. introduced the social factors of users and their behavior into the trust-measuring model, the model effectively reacts to reputation manipulation attack by the malicious users [16]. Based on the Pagerank algorithm, Zhang Yue et al. introduced user behavior feature and the user relational networks, designed a multiple attributes rank (MAR) algorithm to identify influential users [17]. To minimize the influence of the malicious evaluation to the trust model in open distributed network environment, Cai Hongyun et al. proposed a novel trust model based on trust area and evaluation reliability [18]. ZHAO Bin et al. introduced the multiple factors, such as the bonuspenalty factors, evaluation reliability of the recommending entities and balanced weight, into trust evaluation to improve the reliability and accuracy of trust [19].

Although it is less directly related to current work and user reliability computing in the open knowledge organization, the problem that both solve has the same essence, they quantify the reliability of network user behavior and provide more safe and reliable basis for making trusted decision. Therefore, current work has certain referent significance on evaluating the user behavior reliability from the views of multi-attribute and provides a theoretical basis under the open knowledge environment for this paper.

\section{USER RELIABILITY DYNAMIC COMPUTATIONAL METHOD}

\subsection{Related Definition}

Based on the current theory and method of related work, we define the main concepts related to URDCM as follows:

Definition1: Behavior refers to a certain operation of a user participating in knowledge organization.

According to the impact of user behavior on the knowledge organization structure, user behavior includes direct and indirect behavior.

Direct behavior: refers to the behavior except consumption behavior that has direct impact on knowledge organization structure, including new-built, modification, deletion and evaluation of knowledge.

Indirect behavior: refers to the consumption behavior, which has no impact on the knowledge organization structure, such as the user recommendation, extraction, sharing, discussion, communication and answering behavior of knowledge.

Definition 2: Score behavior refers to an operation when a user scores a direct behavior except his own score behavior according to the rules, score value is eand represents the reliability (the right degree) of the direct behavior, eis expressed with fuzzy number because of the fuzziness and randomness of user behavior, $e \epsilon$.

Definition 3: Reliability evaluation level refers to evaluation reliability level of a user or a behavior in the open knowledge organization, they could be different. 
Definition 4: Let a set of behavior $A=\left\{a_{1}, a_{2}, . . a_{i}, \ldots a_{l}\right\}$, the corresponding weight set $w=\left\{w_{1}, w_{2}, . . w_{i}, \ldots w_{l}\right\}$. Behavior constraint is the value range of $i^{\text {th }}$ type $a_{\mathrm{i}}$ of $k$ level user, such as behavior scale range and evaluation value range. constraint range is $\left[\xi_{k i}, \xi_{k i+1}\right), 0 \leq \xi_{k i}<\xi_{k i+1} \leq z$, $\mathrm{z}$ is determined by experts, the same behavior type with different user level may have different constraint.

Definition 5: Group is made of the users participating in knowledge organization in same domain. In this paper, when group and user are presented at the same scene, they belong to the same domain except when a special explanation is provided.

Definition 6: Decision attribute is the index of quantifying user behavior and computing reliability.

According to the characteristic of open knowledge organization, the key point of URDCM is to choose some reasonable decision attributes. Three factors have main influence on the user reliability as follows:

1. User direct behavior: User direct behavior is the basis of open knowledge organization, and reflects the knowledge abilities and the main perspective of the user about domain knowledge. The direct behavior quality is one of the important factors influencing user behavior reliability.

2. Consumption behavior: Consumption behavior is the supplement of direct behavior, which implies the trust assumption among users. Consumption behavior includes group consumption behavior and user consumption behavior. User consumption behavior promotes the improvement of own knowledge ability, which has a positive effect on own behavior reliability at the same time. The higher the group consumption scale on a behavior, the greater the group trust on the behavior.

3. Time factor: A user could have different positivity at different time interval, that is, the behavior scale of user participation in knowledge organization may be different. When a user does not participate or seldom participates in knowledge organization at a time interval, the user positivity is very low, the group will reduce its trust on the user. Therefore, the time factor is an important factor to affect user reliability.

According to the above analysis, this paper will discuss how to qualify user reliability from the decision attributes of user direct reliability, bonus-penalty factor, group consumption factor and user consumption factor combined with time factor.

Definition 7: User direct reliability (UDR) in a domain is integrated by the reliability of user direct behavior $a_{i}, \ldots a_{m}$ in the domain.

Definition 8: User reliability in a domain will be gained by integrating user direct reliability, bonus-penalty factor, group consumption factor and user consumption factor in the domain.

\subsection{Decision Attributes Computing}

\subsubsection{Bonus-Penalty Factor}

The bonus-penalty factor is a decision attribute introduced to adjust user direct reliability, encourage user positivity taking part in knowledge organization and improve the quality of knowledge organization in a time interval.

Bonus-penalty factor is determined by direct behavior positivity. At time $t$, user $u$ belongs to $k$ level user, let his direct behavior scale constraint $\left[\xi_{k i}, \xi_{k i+1}\right]$, at a time interval $\left[t_{j}, t\right], \mathrm{a}_{\mathrm{i}}$ scale is $\Delta x_{j}$. Then, the influence of positivity on reliability of user $u$ direct behavior is described as follows:

If $\Delta x_{j}<\xi_{k i}$ then the user is in negative state, positivity is low, and the user reliability will be reduced;

If $\Delta x_{j}>\xi_{k i+1}$ then user is in positive state, positivity is high, user reliability will be increased at the time interval

If $\xi_{k i} \leq \Delta x_{j} \leq \xi_{k i+1}$ then user is in normal state, positivity is normal, user reliability will not be influenced at the time interval.

Let the use direct reliability of user $u$ and bonus-penalty factor expressed as $s$ and respectively $\varepsilon^{\Delta t j}$. So the function of bonus-penalty factor user $u$ could be expressed as formula (1):

$$
f_{1}\left(u, \Delta x_{j}\right)=\left\{\begin{array}{cc}
1 & \xi_{k i} \leq \Delta x_{j} \leq \xi_{k i+1} \\
1-b_{1}\left(\Delta x_{j}-\xi_{k i}\right) /\left(\Delta x_{j}+\xi_{k i}\right) & \Delta x_{j}<\xi_{k i} \\
1-b_{2}\left(\Delta x_{j}-\xi_{k i+1}\right) /\left(\Delta x_{j}+\xi_{k i+1}\right) & \Delta x_{j}>\xi_{k i+1}
\end{array}\right.
$$


$0<b_{1}, b_{2} \leq 1, b_{1}, b_{2}$ are parameters to adjust bonus-penalty speed, the larger $b_{1}$, the faster user direct reliability drops, the lower $b_{2}$, the faster user direct reliability increases.

Therefore, at time interval $\left[t_{j}, t\right]$ bonus-penalty factor $\varepsilon^{4 t j}$ is defined as follows:

$$
\varepsilon^{\Delta t_{j}}=s^{f_{1}\left(u, \Delta x_{j}\right)}-s
$$

At time $t$ bonus-penalty factor $\varepsilon^{t}$ is defined as follows:

$$
\begin{aligned}
& \varepsilon^{t}= \begin{cases}\mu & \mu_{1}<u<u_{2} \\
\mu_{1} & u \leq u_{1} \\
\mu_{2} & u \geq u_{2}\end{cases} \\
& u=\sum_{i=1}^{j} \varepsilon^{\Delta t_{j}}
\end{aligned}
$$

$-1 \leq \mu_{1}<0,0 \leq \mu_{2}<1, \mu_{1}$ is minimum value of bonus-penalty factor, $\mu_{2}$ is a maximum of bonus-penalty factor.

\subsubsection{Consumption Factor}

Consumption factor is a decision attribute introduced to indicate the influence of group consumption behavior and user consumption behavior on user reliability, to raise the quality of user direct behavior, and to promote the communication and knowledge shared among users. Thus, consumption factor includes group consumption factor and user consumption factor. Group consumption factor is used to modify single direct behavior reliability and user consumption factor is used to revise user direct reliability.

Suppose consumption scale is larger than the threshold value that means it will have positive effect on user reliability. Let consumption scale constrain of $k$ level user be $\left[u C_{k}, \infty\right)$, group consumption scale constrain to $a_{i}$ be $\left[G C_{K i} \infty\right)$. At time $t$, user $u$ belongs to $k$ level user, his consumption scale is, and group consumption scale on $a_{i}$ is $y_{j}$. To avoid malicious consumption and promote communication among users, group consumption factor to $a_{i}$ and user $u$ consumption factor $f_{2}\left(\mathrm{a}_{\mathrm{i}}, \mathrm{y}_{\mathrm{j}}\right)$ are defined $f_{3}\left(u, z_{j}\right)$ as formula (3) and (4):

$$
\begin{gathered}
f_{2}\left(a_{i}, y_{j}\right)=\left\{\begin{array}{cc}
1 & y_{j} \leq G C_{k j} \\
b_{3}-b_{3} /\left(1+\sqrt[3]{y_{j}}\right) & y_{j}>G C_{k j}
\end{array}\right. \\
f_{3}\left(u, z_{j}\right)= \begin{cases}1 & z_{j} \leq u C_{k} \\
b_{4}-b_{4} /\left(1+\sqrt[3]{z_{j}}\right) & z_{j}>u C_{k}\end{cases}
\end{gathered}
$$

$0<, b_{3}, b_{4} \leq 1$ parameter $b_{3}$ and $b_{4}$ are the adjusting parameters, the smaller the $b_{3}$ and $b_{4}$, the larger the speed of consumption factor adjusting user reliability .

\subsubsection{User Direct Reliability}

At time $t, G=\left\{u_{1}, u_{2}, \ldots u_{i} \ldots u_{n}\right\}$ set means $n$ autonomous users in current open knowledge organization, the corresponding reliability set $C=\left\{c_{1}, c_{2}, . . c_{i}, \ldots c_{n}\right\} ; u$ is the current user $u \in G$, and direct behavior set $A=\left\{a_{1}, a_{2}, . . a_{i}, \ldots a_{m}\right\}$. is scored, comprehensive score value set of $\mathrm{G}$ on a $\mathrm{a}_{\mathrm{i}}$ is $E_{i}=\left\{e_{i l}, e_{i 2}, \ldots, a_{i n}\right\}$. The comprehensive evaluation set $A$ of $G$ is $G E=\left\{G e_{1}, G e_{2}, \ldots, G e_{m}\right\}$, the score value set of $u$ on $A$ is, $u E=\left\{e_{1}, e_{2}, \ldots, e_{m}\right\}, e_{i} \in E_{i}$.

User direct reliability (UDR) is integration of all of the single direct behavior reliability of user, not only related to group evaluation behavior but also user consumption behavior and user score consumption. Hence, we will discuss the single direct behavior reliability (single DBR) first.

\section{(1) Single Direct Behavior Reliability}

There may be a large number of unreliable and low quality scores in the process of open knowledge organization, group comprehensive score $G E_{i}$ represents group common opinion on $a_{i}, a_{i} \in A$. Therefore, we think $a_{i}$ single DBR computational method includes three steps: 
Step 1, computing $G e_{i}$;

Step 2, computing group score reliability $G e_{i}^{\prime}$; by removing the low and unreliable scores;

Step 3, using group consumption factor revising group score reliability.

is the $e_{i j}$ score on $a_{i}$ of $j^{\text {th }}$ user among $G, G_{e i}$ is expressed with weighted average method as follows:

$$
G e_{i}=\sum_{j=1}^{n} e_{i j}\left(c_{i} / \sum_{k=1}^{n} c_{i}\right)
$$

We can let threshold value $h$ about $\left|e_{i j}-G e_{i}\right|$ to remove unreliable and low quality scores. If $\left|e_{i j}-G e_{i}\right|>h$, then the score $e_{i j}$ is unreliable, and does not take part in compute $a_{1}$ group score reliability. If $\left|e_{i j}-G e_{i}\right| \leq h$, then the score $e_{i j}$ is useful to compute $a_{i}$ group score reliability.

Let useful score set $E^{\prime}$ of $G$ on $a_{i}\left(e_{i 1}^{\prime} \ldots, e_{n}^{\prime}\right)$, and corresponding user reliability is $\left(c_{1}^{\prime} \ldots, c_{n}^{\prime}\right)$, the a $a_{i}$ group scorere liability $G e_{i}^{\prime}$ could be expressed as follows:

$$
G e_{i}^{\prime}= \begin{cases}G e_{i} & n<m_{\min }, \frac{n^{\prime}}{n}<n_{0} \\ \sum_{i=1}^{n^{\prime}} e_{i j}^{\prime}\left(c_{j}^{\prime} / \sum_{j=1}^{n^{\prime}} c_{j}^{\prime}\right) & \left|e_{i j}^{\prime}-G e_{i}\right| \leq h, m_{\min } \leq n \leq m_{\max }, n_{0} \leq \frac{n^{\prime}}{n} \leq 1\end{cases}
$$

$n_{0}$ is threshold value, controlling the minimum of useful behavior scale $n^{\prime}$ at computing value to ensure that $G e_{i}^{\prime}$ represents the opinions of most users among $G, m_{\min }$ and $m_{\max }$ are the minimum and maximum of $n$ at computing $G e_{i}$ group useful scale respectively. If $n$ exceeds, $m_{\max }$ and group does not achieve common opinions $a_{i}$ will be submitted to domain experts who will confirm $G e_{i}^{\prime}$ value.

Group consumption has a positive impact on $a_{i}$ reliability, so $a_{i}$ single DBR is expressed by group consumption factor score revising reliability as follows:

$$
G a_{j}=\left(G e_{i}^{\prime}\right)^{f_{2}\left(a_{i}, y_{i}\right)}
$$

\section{(2) User Score Behavior Reliability}

User score expresses the opinion and evaluation value on single direct behavior, and cannot express the score behavior reliability (SBR). Single DBR represents opinions of most users, has a higher reference value for user SBR, and so the evaluation similarity between group and a user will be used to indicate user score reliability in this paper. If two sets of evaluation values have high evaluation similarity, in other word, the closer the evaluation distance, the higher the user SBR .

Let user $u$ score value $a_{i}, e_{i}$ so the evaluation distance between user $u$ and group $G$ is expressed as follows:

$$
\operatorname{dis}\left(u, G, a_{i}\right)=\left|e_{i 0}-G a_{i}\right|
$$

In open knowledge system, there are two states for behavior $a_{i}$ : certainty (reliability, unreliability) and uncertainty, suppose $a_{i}$ is in certainty, then

- If the evaluation value between $u$ and $G$ about $a_{i}$ is consistent, user $u$ score behavior is reliable, $\operatorname{dis}\left(u, G, a_{i}\right)=0$;

- If the evaluation value between $u$ and $G$ about $a_{i}$ is inconsistent, user $u$ score behavior is unreliable, $\operatorname{dis}\left(u, G, a_{i}\right)$ $=1$;

At time $t$, let direct behavior scale $n, n_{l}$ certain behavior has consistent evaluation value between $u$ and $G$ and uncertain behavior scale is $n_{2}$. The evaluation distance of certain behavior has no fuzziness, so $\left(n-n_{2}\right)$ certain behaviorreliability is represented by pass rate of certain behavior as follows:

$$
s_{1}^{t}=n_{1} /\left(n-n_{2}\right)
$$


Then the pass rate of user reliable behavior $P R$ is expressed as formula:

$$
\begin{gathered}
P R=p^{*} s_{1}{ }^{t}=n_{1} / n \\
p=\left(n-n_{2}\right) / n
\end{gathered}
$$

$p$ is the certainty rate of user direct behavior.

Evaluation distance and similarity are the different representation of the characteristic of user and group evaluation, so similarity is used to represent uncertain behavior reliability, according to [20], similarity to $a_{i}$ is expressed as follows:

$$
g s^{t}\left(a_{i}\right)=\frac{a}{a+\operatorname{dis}\left(u, G, a_{i}\right)}
$$

Parameter $a$ is the evaluation distance value when the similarity is 0.5 . The range of $g s^{t}\left(a_{i}\right)$ is $[\mathrm{a} /(\mathrm{a}+1), 1], g s^{t}\left(a_{i}\right)$ and is standardized as follows:

$$
s^{\prime}\left(a_{i}\right)=\left(g s^{t}\left(a_{i}\right)-(a / a+1)\right) /(1-(a / a+1))
$$

Therefore, user uncertain score behavior reliability $s_{2}^{t}$ is gained by the weighted average method as follows:

$$
s_{2}^{t}=\sum_{i=1}^{n_{2}} w_{i} s^{\prime}\left(a_{i}\right)
$$

At time $t$, UDR is comprehensive reliability of certain and uncertain behavior and expressed as follows:

$$
s^{t}=p s_{1}^{t}+(1-p) s_{2}^{t}
$$

\subsubsection{User Comprehensive Reliability}

User comprehensive reliability is acquired by revising UDR with user consumption factor and bonus-penalty factor; because consumption factor has positive impact on user reliability, at time $t$, user comprehensive reliability is expressed as follows:

$$
C_{u}{ }^{t}= \begin{cases}\left(s^{t}+\varepsilon^{t}\right)^{f_{3}\left(u, z_{j}\right)} & 0<s^{t}+\varepsilon^{t} \leq 1 \\ 0 & s^{t}+\varepsilon^{t} \leq 0\end{cases}
$$

\section{STIMULATION ANALYSIS}

Now, the open knowledge organization system mainly records users' contribution behavior to knowledge organization (creation and edition) without dividing group behavior into different domains, and does not record the behavior of user consumption too. Moreover, there is uncertainty and randomness for user behavior, therefore the data of user and group behavior will be generated with the stochastic simulation method, and then URDCM is simulated and analyzed to verify its feasibility. Because $a_{i}$ group score reliability, user $u$ SBR and the influence of bonus-penalty factor on user DBR are keys to realize the URDCM, we will focus on three experiments that simulate the changes of the process of user reliability in different and continuous time intervals under group or user behavior. The whole simulation process is divided into continuous and equal time intervals.

In order to be close to reality and simplify the experiment, we suppose the role and behavior of users are independent of each other in the experiment. Most common behavior types are directly chosen in open knowledge system, for example, direct behavior includes new-built, modification and evaluation, and consumption behavior includes share, recommendation, extraction and download and so on. All kinds of direct behavior and consumption behavior are in the same position and have same weight, the stimulated users are ordinary user, and the relevant parameters of reliability are shown in Tables $\mathbf{1}, \mathbf{2}$.

\section{Experiment 1}

It aims at verifying the sensibility of $a_{i}$ group score reliability on low-quality score behavior during continuous time 
intervals when group useful score scale is introduced into computing UDR, comparing with group comprehensive score.

In the experiment, group participating in scores are ordinary users and have same reliability, $m$ is group scale, $a_{i}$ group scores are randomly generated in five continuous time intervals, and the detail is following:

$$
\begin{aligned}
& t_{0} \sim t_{1}:\{1.0000,0.1233,0.0134,0.3697,0.6986,0.8893,0.5938,0.1567,0.8214,0.9501\} \\
& t_{1} \sim t_{2}:\{0.0084,0.3969,0.6499,0.8913,0.7688\} \\
& t_{2} \sim t_{3}:\{0.9355,0.7138,0.9776,0.6371,0.9169,0.2376\} \\
& t_{3} \sim t_{4}:\{0.9697,0.7148,0.782,0.9000\} \\
& t_{4} \sim t_{5}:\{0.8481,0.8021,0.6683,0.671,0.8206,0.7621,0.5000\}
\end{aligned}
$$

Table 1. Reliability level of behavior.

\begin{tabular}{|c|c|}
\hline Reliability range & Level \\
\hline$(0,0.4)$ & unreliable \\
\hline$(0.4,0.6)$ & basic reliable \\
\hline$(0.6,0.8)$ & reliable \\
\hline$(0.8,0.95)$ & very reliable \\
\hline$(0.95,1)$ & eminently reliable \\
\hline
\end{tabular}

Table 2. User level, reliability range and behavior scale constrains.

\begin{tabular}{|c|c|c|c|}
\hline Level & Name & Reliability range & {$\left[\xi_{k i}, \xi_{k i+1}\right]$} \\
\hline 1 & ordinary user & $(0,0.7)$ & $(4,12)$ \\
\hline 2 & advanced user & $(0.7,0.8)$ & $(12,50)$ \\
\hline 3 & excellent user & $(0.8,0.9)$ & $(30,80)$ \\
\hline 4 & Expert & $(0.9,0.95)$ & $(70,100)$ \\
\hline 5 & advanced expert & $(0.95,1)$ & $(90,120)$ \\
\hline
\end{tabular}

According to the computational method of $a_{i}$ group score reliability, at time $t, a_{i}$ group score reliability $G e_{i}$ and group comprehensive score $G e_{i}^{\prime}$ in continuous interval times is shown in Table 3.

Table 3. $a_{i}$ group score reliability and group comprehensive score at time $t$.

\begin{tabular}{|c|c|c|c|c|c|}
\hline Reliability & $\boldsymbol{t 1}$ & $\boldsymbol{t} \mathbf{2}$ & $\boldsymbol{t 3}$ & $\boldsymbol{t 4}$ & $\boldsymbol{t 5}$ \\
\hline & 0.5616 & 0.5554 & 0.6032 & 0.6447 & 0.6622 \\
\hline$G e_{i}^{\prime}(h=0.3, n 0=0.5)$ & 0.5616 & 0.5554 & 0.6755 & 0.7300 & 0.7376 \\
\hline$G e_{i}^{\prime}(h=0.3, n 0=0.6)$ & 0.5616 & 0.5554 & 0.6032 & 0.7300 & 0.7376 \\
\hline$G e_{i}^{\prime}(h=0.35, n 0=0.5)$ & 0.6746 & 0.6755 & 0.7309 & 0.7672 & 0.7648 \\
\hline$(h=0.35, n 0=0.6)$ & 0.5616 & 0.6755 & 0.7309 & 0.7672 & 0.7648 \\
\hline$n^{\prime} / n(h=0.3)$ & $4 / 10$ & $7 / 15$ & $11 / 21$ & $16 / 25$ & $24 / 32$ \\
\hline$n^{\prime} / n(h=0.35)$ & $5 / 10$ & $9 / 15$ & $14 / 21$ & $19 / 25$ & $27 / 32$ \\
\hline
\end{tabular}

From Table 3, with group score scale increasing, group are coming to common opinion and $a_{i}$ reliability shows the characteristic of growth trend, $G e_{i}^{\prime} \geq G e_{i}$ and $G e_{i}^{\prime}$, removes part of low quality score.

With score scale increasing, the values of parameter $h$ and $n_{0}$ are different, the value of $n$ is different, so the number of $G e_{i}^{\prime}$ removing low quality score is different If, $n^{\prime} / n<n_{0}$ this indicates that group scores are disperse, and the majority of group scores are unusual and will be removed, if so, only represents the opinions of minority of group, $G e_{i}^{\prime}$ so is degraded to $G e_{i}$. For example, $G e_{i}^{\prime}(h=0.35, n=0.6)$ at time $t_{l}, G e_{i}^{\prime}(h=0.3, n=0.5)$ at time $t_{1}$ and $t_{2}, G e_{i}^{\prime}(h=0.3, n=0.6)$ at time $t_{1}, t_{2}$ and $t_{3}, G e_{i}^{\prime}$ is equal to $G e_{i}$, If $n^{\prime} / n \geq n_{0} \mathrm{Ge}_{\mathrm{i}}^{\prime}$ represents the opinions of majority of group, and few scores of group are removed. Therefore, $G e_{i}^{\prime}$ can more efficiently represent the influence of group score behavior on $\mathrm{a}_{\mathrm{i}}$ than that of $G e_{i}^{\prime}$. 


\section{Discussion}

Generally, the larger the threshold $h$ and the smaller the $n$, the better $G e_{i}^{\prime}$ can represent the opinions of the majority of group. However, if $h$ is equal to 1 and is equal to, $G e_{i}^{\prime}$ will represent the opinions of all users of group and unusual scores cannot be removed; if the smaller is threshold $h$ and the larger is $n, G e_{i}^{\prime}$ may only represent the opinions of minority of group. In two cases, is degraded to, therefore, $h$ and value need to be determined according to the actual demand.

\section{Experiment 2}

It aims at verifying the influence of uncertain behavior on user SBR in continuous time intervals when uncertain behavior reliability is introduced to user reliability computing, compared with pass rate $(P R)$ of user reliable behavior.

In the experiment, suppose the number of user certain behavior is unchangeable in continuous time intervals, and the number of user uncertain behavior is increasing. At time $t$, let certain direct behavior scale be $n_{1}$, and $n_{3}$ is certain direct behavior approved by the group. Uncertain behavior scale is $n_{2}$. Suppose during following five continuous time intervals, $n_{l}$ and $n_{3}$ stay unchangeable, that is, there is no behavior achieving common opinion by the group, based on which, the influence of uncertain behavior on user reliability is simulated. Let parameter $n=30, n_{3}=20$ and $n_{2}=6$, then $s_{I}=0.8333$. The behavior set $A=\left\{a_{1}, a_{2}, \ldots, a_{n}\right\}$ is the common score object of user $u$ and group, the score sets of user $u$ and group are $\left\{e_{j 0}\right\}$ and $\left\{G \mathrm{a}_{\mathrm{j}}\right\}$ respectively, and randomly generated. respectively, and randomly generated. The detail is shown in Table 4.

Table 4. Uncertain behavior score of user $u$ and group.

\begin{tabular}{|c|c|c|c|}
\hline \multicolumn{2}{|c|}{ Time interval } & \multirow[b]{2}{*}{$0.2731,0.8656,0.8049,0.2319,0.0498,0.6408$} & \multirow{2}{*}{$\frac{\boldsymbol{n}}{6}$} \\
\hline $\mathrm{t} \sim \mathrm{t}_{1}$ & $\left\{e_{j 0}\right\}$ & & \\
\hline & $\left\{G \mathrm{a}_{j}\right\}$ & $\{0.2548,0.2324,0.9084,0.2393,0.0784,0.1909\}$ & \\
\hline \multirow[t]{2}{*}{$t_{1} \sim t_{2}$} & $\left\{e_{j 0}\right\}$ & $\{0.8439,0.1708,0.4398,0.3142,0.3932,0.1197,0.4586,0.9342,0.1603,0.2379,0.9669,0.8704,0.137\}$ & 13 \\
\hline & $\left\{G \mathrm{a}_{j}\right\}$ & $\{0.1739,0.9943,0.34,0.3651,0.5915,0.0381,0.8699,0.2644,0.8729,0.6458,0.6649,0.0099,0.8188\}$ & \\
\hline \multirow[t]{2}{*}{$\mathrm{t}_{2} \sim \mathrm{t}_{3}$} & $\left\{e_{j 0}\right\}$ & $\{0.4302,0.7349,0.3461,0.1556\}$ & 4 \\
\hline & $\left\{G \mathrm{a}_{j}\right\}$ & $\{0.8903,0.6873,0.166,0.1911\}$ & \\
\hline \multirow[t]{2}{*}{$t_{3} \sim t_{4}$} & $\left\{e_{j}\right\}$ & $\{0.4225,0.4902,0.4608\}$ & 3 \\
\hline & $\left\{G \mathrm{a}_{j}\right\}$ & $\{0.856,0.8159,0.4574\}$ & \\
\hline \multirow[t]{2}{*}{$t_{4} \sim t_{5}$} & $\left\{e_{j 0}\right\}$ & $\{0.4507,0.9016,0.2974,0.6932\}$ & 4 \\
\hline & $\left\{G \mathrm{a}_{i}\right\}$ & $\{0.4122,0.0056,0.0492,0.6501\}$ & \\
\hline
\end{tabular}

Because user uncertain behavior reliability varies in differentvalue of parameter $a$, the experiment chooses five different values for parameter $a$, that is $0.1,0.2,0.3,0.4$ and 0.5 . The reliabilities of uncertain behavior $s_{2}$ and UDR $s$ are shown in Tables $\mathbf{5}, \mathbf{6}$ respectively.

Table 5. User $u$ uncertain reliability at time $t$.

\begin{tabular}{|c|c|c|c|c|c|}
\hline Reliability & $\boldsymbol{t}_{\boldsymbol{1}}$ & $\boldsymbol{t}_{2}$ & $\boldsymbol{t}_{\boldsymbol{3}}$ & $\boldsymbol{t}_{\boldsymbol{4}}$ & $\boldsymbol{t}_{\boldsymbol{5}}$ \\
\hline$s_{2}(a=0.5)$ & 0.6730 & 0.4606 & 0.4959 & 0.5041 & 0.5140 \\
\hline$s_{2}(a=0.4)$ & 0.6555 & 0.4392 & 0.4738 & 0.4819 & 0.4922 \\
\hline$s_{2}(a=0.3)$ & 0.6309 & 0.4102 & 0.4436 & 0.4517 & 0.4623 \\
\hline$s_{2}(a=0.2)$ & 0.5924 & 0.3674 & 0.3984 & 0.4069 & 0.4175 \\
\hline$s_{2}(a=0.1)$ & 0.5167 & 0.2924 & 0.3174 & 0.3281 & 0.3373 \\
\hline
\end{tabular}

From Table 6, the scale of uncertain behavior is increasing, and $P R$ and $s$ value are descending, but the $s$ value is greater than the value $R$ at time $t_{i} \sim t_{5}$. It is known that group has not come to an agreement on behavior from the Table $\mathbf{5}$, but the highly consistent uncertain behavior score between user $u$ and group exists at five continuous time intervals. For example, at time intervals $t \sim t_{l}$, the uncertain behavior score is more consistent between user $u$ and group, $\mathrm{s}_{2}>0.5$, and uncertain behavior influence on user $u$ reliabilityis considered in computing $s$ value and fails to take into account the computing $P R$ value. So if there exists highly consistent uncertain behavior between user $u$ and group, then $s>P R$, otherwises $=P R$. 
Table 6. User $u$ direct reliability at time $t$.

\begin{tabular}{|c|c|c|c|c|c|}
\hline Reliability & $\boldsymbol{t}_{\boldsymbol{1}}$ & $\boldsymbol{t}_{\boldsymbol{2}}$ & $\boldsymbol{t}_{\boldsymbol{3}}$ & $\boldsymbol{t}_{\boldsymbol{4}}$ & $\boldsymbol{t}_{\boldsymbol{5}}$ \\
\hline$s(a=0.5)$ & 0.8013 & 0.7163 & 0.6972 & 0.6788 & 0.6560 \\
\hline$s(a=0.4)$ & 0.7978 & 0.7069 & 0.6864 & 0.6673 & 0.6438 \\
\hline$s(a=0.3)$ & 0.7929 & 0.6941 & 0.6716 & 0.6516 & 0.6272 \\
\hline$s(a=0.2)$ & 0.7852 & 0.6751 & 0.6495 & 0.6283 & 0.6023 \\
\hline$s(a=0.1)$ & 0.7700 & 0.6420 & 0.6098 & 0.5873 & 0.5578 \\
\hline$P R$ & 0.6667 & 0.5128 & 0.4545 & 0.4167 & 0.3704 \\
\hline
\end{tabular}

In a word, uncertain behavior reliability introduced into user reliability computing will more fully represent user behavior reliability, especially if $p=0$, then $s^{=} s_{2}, P R=0$.

\section{Discussion}

According to Table 5, at time $t$, uncertain behavior reliability $s_{2}$ of user $u$ is increasing with the increase in parameter $a$, because parameter $a$ decides the maximum score distance $d$ of between user $u$ and group whether a behavior is reliable, the smaller parameter $a$ valueis, the smaller $d$ value is. So parameter $a$ value is determined to meet the open knowledge organization demand.

\section{Experiment 3}

The experiment aims at verifying the influence of user comprehensive reliability during continuous time intervals after the bonus-penalty factor is introduced into URDCM. The experiment will observe the influence of user direct behavior scale on UDR in continuous interval times.

Let parameter $b_{1}=b_{2}=0.1, a=0.3, \mathrm{u}=0.1$ the data is the same as experiment two, and the UDR $s$ value is equal to $s$ $(a=0.3)$. The detail is shown in Table 7 .

From Table 7 it can be seen that user reliability is different at time $t_{1}, t_{2}, t_{3}, t_{4}$ and $t_{5}$, user level evolves from ordinary user to excellent user and then excellent degenerates into advanced user, and advanced user degenerates into ordinary user.

Table 7. User Reliability introducing the bonus-penalty factor.

\begin{tabular}{|c|c|c|c|c|c|}
\hline Type & $\mathbf{t} \sim \mathbf{t} 1$ & $\mathbf{t 1} \sim \mathbf{t} \mathbf{t}$ & $\mathbf{t 2 \sim t 3} \sim \mathbf{t 4}$ & $\mathbf{t 4 \sim t 5}$ \\
\hline$\Delta \mathrm{x}_{\mathrm{j}}$ & 6 & 13 & 4 & 3 & 4 \\
\hline$s(a=0.3)$ & 0.8099 & 0.7542 & 0.7344 & 0.7174 & 0.6962 \\
\hline$C_{u}^{*}$ & 0.8099 & 0.7542 & 0.7232 & 0.6929 & 0.6937 \\
\hline$\varepsilon$ & 0 & 0 & $\mathbf{- 0 . 0 1 1 2}$ & $\mathbf{- 0 . 0 2 4 5}$ & $\mathbf{- 0 . 0 2 4 5}$ \\
\hline$P R$ & 0.6667 & 0.5128 & 0.4545 & 0.4167 & 0.3704 \\
\hline $\begin{array}{c}\text { User level } \\
s(a=0.3)\end{array}$ & excellent user & $\begin{array}{c}\text { advanced } \\
\text { user }\end{array}$ & $\begin{array}{c}\text { advanced } \\
\text { user }\end{array}$ & $\begin{array}{c}\text { ordinary } \\
\text { user }\end{array}$ & $\begin{array}{c}\text { ordinary } \\
\text { user }\end{array}$ \\
\hline
\end{tabular}

Different levels of users have different positivity constrains. According to Table $\mathbf{1}$, at time intervals $t_{2} \sim t_{3}$ and $t_{3} \sim t_{4}$, user positivity is lower, therefore, at time $t_{3}$ and $t_{4},<0$, user reliability is modified through the bonus-penalty factor, and $C_{u}^{t}<s$, and the level of user $u$ is degenerated from advanced user into ordinary user.

Although user positivity is normal and user direct behavior has no influence on the user reliability at time interval $C_{u} t_{4} \sim t_{5}$, it is still affected by user positivity at time intervals $t_{2} \sim t_{3}$ and $t_{3} \sim t_{4}$, and revised by the bonus-penalty factor $\varepsilon$. Similarly, if $\varepsilon>0, C_{u}^{t}$ is affected by user positivity too, and $C_{u}^{t} \geq \mathrm{s}$. So comparing with $P R$, the method can accurately and dynamically reflect the changes of user behavior positivity in the process of open knowledge organization and then better improve the enthusiasm of user participating in knowledge organization.

\section{CONCLUSION}

The paper puts forward a dynamic computational method of user reliability, which quantifies user reliability from the perspective of multi-attribute such as direct reliability, bonus-penalty factor, and consumption factor.

This method can more comprehensively express user behavior reliability and improve user enthusiasm participating 
in knowledge organization and the quality of ontology, and promote consumption behavior positivity including knowledge learning, communication and sharing between users.

\section{CONFLICT OF INTEREST}

The authors confirm that this article content has no conflict of interest.

\section{ACKNOWLEDGEMENTS}

The research work was supported by General Research for Humanities and Social Sciences Project, and Chinese Ministry of Education (Grant No. 11YJA870007), and the Fundamental Research Funds for the Central Universities (Grant No. 2013B09314, 2015B28014 and 2015B23914), P.R. China, and the Research Innovation Program for College Graduates of Jiangsu Province (Grant No. KYLX15_0523).

\section{REFERENCES}

[1] G.J. Xian, and R.X. Zhao, "A review and prospects on collaborative ontology editing tools", J. Integr. Agr, vol. 11, pp. 731-740, 2012. [http://dx.doi.org/10.1016/S2095-3119(12)60062-8]

[2] W.C. Xie, and J.M. Yang, "Empirical analysis Wikipedia contributors of human dynamics", Stud. Sci. Sci., vol. 28, pp. 1454-1458, 2010.

[3] R. Priedhorsky, and J. Chen, Creating, Destroying, and Restoring Value in Wikipedia. The 2007 International ACM Conference on Supporting Group Work, 2007, pp. 259-268. [http://dx.doi.org/10.1145/1316624.1316663]

[4] T. Tudorache, C. Nyulas, N.F. Noy, and M.A. Musen, "WebProtégé: A collaborative ontology editor and knowledge acquisition tool for the web", Semant. Web, vol. 4, no. 1, pp. 89-99, 2013. [PMID: 23807872]

[5] J. Bao, and V. Honavar, "Collaborative ontology building with Wiki@nt - a multi-agent based ontology building environment", In: Proceedings of the $3^{\text {rd }}$ International Workshop on Evaluation of Entology Based Tools. Hiroshema, Japan, 2004. Available from: http://ceur-ws.org/Vol-128/EON2004_Baojie.pdf

[6] S. Auer, S. Dietzold, and T. Riechert, "OntoWiki-A tool for social, semantic collaboration", In: The Semantic Web-ISWC 2006. $5^{\text {th }}$ International Semantic Web Conference, vol. 4273. ISWC, 2006, pp. 736-749.

[7] Z.C. Luo, W.Q. Guan, and L. Zhang, "Comparative analysis in Wiki and Baidu Baike", Inf. Stud. Theory Appl., vol. 32, pp. 71-74, 2009.

[8] L.Y. Wan, "Research on the interaction mechanism in online encyclopedia users' collaborative authoring take Baidu Baike post bar for example", J. Intell., vol. 33, pp. 167-172, 2014.

[9] J. Chang, and R. Q. Ou, Study on the Prompting of Peer Producers in Baidupedia. Science \& Technology and Economy, 2014 , pp. 81-85.

[10] L.H. Huang, Q.H. Zhu, and C. Shen, "The dynamic characteristics of Wiki users contribution behavior based on multi-agent", J. China Soc. Sci. Tech. Info., vol. 33, pp. 97-112, 2014.

[11] X.Y. Li, and X.L. Gui, "Cognitive model of dynamic trust forecasting", J. Softw., vol. 21, pp. 163-176, 2010. [http://dx.doi.org/10.3724/SP.J.1001.2010.03558]

[12] L. L. Song, R.F. Wang, and H. B. Zhang, "Service reputation modeling and user reliability evaluation calculating", J. WUT, Infor. Manag. Eng., vol. 35, pp. 452-455, 2013.

[13] L.V. You, J.Z. Luo, and W. Li, "Adaptive user behavior's evaluation method based on network status", J. Commun., vol. 34 , pp. 71-80, 2013.

[14] Q.H. Liu, X.N. Wu, and L. Yang, "Weighted trust computation method based on behavior of users", J. Comp. Appl., vol. 31, pp. 1887-1890, 2011.

[15] Z. Jiang, S.Q. Li, and C.G. Yin, "Evaluating network user behavior trust based on multiple decisions attributes", Jisuanji Yingyong Yanjiu, vol. 28, pp. 2289-2293, 2320, 2011.

[16] L.V. You, Z. Hua, and H. Sheng, "Trust measuring model based on social factors of users and their behavior", Comput. Sci., vol. 40, pp. 127-131, 2013.

[17] Y. Zhang, H.L. Zhang, and W.Z. Zhang, "Identifying the influential users in network forum", J. Comp. Res. Devel., vol. 50, pp. 2195-2205, 2013.

[18] H.Y. Cai, J. F. Tian, and Z. Li, "Trust model based on trust area and evaluation reliability", J. Comp. Res. Devel., vol. 50, pp. 2138-2131, 2011.

[19] B. Zhao, J.S. He, and Y.X. Zhang, "Analysis of multi-factor in trust evaluation of open network", J. Shandong Univ. Nat. Sci., no. September, pp. 103-108, 2014. 
[20] K.B. Wei, Y.P. Ran, and N. Yu, "The research and analysis of computing methods on semantic similarity", Comp. Tech. Devel., vol. 20, pp. $102-105,2010$

(C) Feng et al.; Licensee Bentham Open.

This is an open access article licensed under the terms of the Creative Commons Attribution-Non-Commercial 4.0 International Public License (CC BY-NC 4.0) (https://creativecommons.org/licenses/by-nc/4.0/legalcode), which permits unrestricted, non-commercial use, distribution and reproduction in any medium, provided the work is properly cited. 